\title{
La sustentabilidad: una poderosa idea para la humanidad
}

\author{
Sustainability: a powerful idea for humanity
}

Plinio Zarta Avila*

Citar este artículo como: Zarta Avila, Plinio. "La sustentabilidad: una poderosa idea para la humanidad", Revista nodo, 12(22). Bogotá, 2017, pp. 58-67

\section{Resumen}

La complejidad de fenómenos y la variedad de relaciones que involucran la sustentabilidad, como por ejemplo la supervivencia de millones de especies vivas en el planeta (así como también la desaparición de otras tantas) y su relación con los ecosistemas de la naturaleza y de estos con la sociedad, en mi condición de Economista me ha llevado a preguntarme: .cual es el nuevo rol de la economía dentro de la sociedad? Este interrogante me ha motivado a estudiar y a escribir algunas líneas iniciales que deseo ir afinando, hasta alcanzar una mayor profundidad en el tema para presentarles mis opiniones a consideración de los lectores, hasta lograr la dinámica de la discusión que amerita el apasionamiento entre lo académico, lo cognoscitivo y lo sensorial en la vida que llevamos.

Palabras clave: complejidad, sustentabilidad, economía sostenible

\section{Abstract}

The complexity of phenomena and the variety of relationships that involve sustainability, such as the survival of millions of living species on the planet (as well as the disappearance of many others) and their relationship with the ecosystems of nature and of these with Society, as an Economist, has led me to ask myself: what is the new role of the economy within society? This question has motivated me to study and write some initial lines that I want to refine, until reaching a greater depth in the subject to present my opinions to the consideration of the readers, until achieving the dynamics of the discussion that deserves the passion between the academic, the cognitive and the sensory in the life we lead.

Keywords: complexity, sustainability, sustainable economy

Fecha de recepción: 22 de febrero de 2017 • Fecha de aceptación: 5 de junio de 2017

\footnotetext{
* Economista de la Universidad Nacional de Colombia, con Magister en Ciencias Económicas. Área de profundización: Economía y Gestión Pública de la Universidad Nacional de Colombia. Me he desempeñado profesionalmente desde 1986 en diferentes instituciones públicas y privadas, liderando importantes funciones como Gestor, Director, Administrador, Consultor, Investigador y Docente, en temas relacionados con Hacienda Pública, Análisis Financiero, Comercio Exterior y Desarrollo Económico Local.
} 


\section{Introducción}

Para mediados de 2017, uno de los temas relevantes para la humanidad en el desarrollo de la sociedad en sus diferentes determinaciones (ambientales, económicas y sociales), sigue siendo el relacionado con la sustentabilidad y/o sostenibilidad de la misma, 30 años después de haber aparecido el Informe de las Naciones Unidas en 1987, denominado "Informe Brundtland", conocido como Nuestro Futuro Común (ONU, 1988).

El uso indiscriminado al término "sostenible" ha generado un agotamiento de su acepción inicial ya que, según los mejores cánones del marketing futurista, hoy en día todo es sostenible, término que goza de buena aceptación social y está muy relacionado con todo aquello que perdure en el tiempo. Es por esta y otras razones que más adelante contemplo, que prefiero denominarlo sustentable, porque este término, a más de su aspecto temporal, incluye conceptos que interrelacionan aristas muy importantes que preocupan a todos los seres de la tierra, como quiera que tienen que ver con la vida humana, principio y esencia del ser, guardando relación con el presente y futuro del planeta tierra y su interacción con ella, y con base en estos planteamientos, la edificación de una nueva cultura del individuo dentro de la sociedad.

La complejidad de fenómenos y la variedad de relaciones que involucran la sustentabilidad, como por ejemplo la supervivencia de millones de especies vivas en el planeta (así como también la desaparición de otras tantas) y su relación con los ecosistemas de la naturaleza y de estos con la sociedad, en mi condición de Economista me ha llevado a preguntarme: ¿cuál es el nuevo rol de la economía dentro de la sociedad? Este interrogante me ha motivado a estudiar y a escribir algunas líneas iniciales que deseo ir afinando, hasta alcanzar una mayor profundidad en el tema para presentarles mis opiniones a consideración de los lectores, hasta lograr la dinámica de la discusión que amerita el apasionamiento entre lo académico, lo cognoscitivo y lo sensorial en la vida que llevamos.
Como profesor universitario de Economía, comprendí que uno de los temas centrales de la "contemporaneidad" es el relacionado con la "sustentabilidad".

Partiendo de la idea de que lo sustentable contempla valores que deberían ser intrínsecos con nuestro comportamiento, como el poder comprender la limitación de recursos escasos de una sociedad ante unas necesidades humanas diversas e ilimitadas y su relación con los límites de crecimiento económico; como la necesidad de transformar el sistema económico dominante para garantizar que la industria y la agricultura produzcan energías limpias sobre la base de la utilización de recursos renovables; como la satisfacción de las necesidades presentes sin comprometer las generaciones futuras, con el propósito de encontrar el bien común; todos estos fundamentos de lo "sustentable" traen consigo un nuevo enfoque sobre la necesidad de un cambio en la mentalidad humana, a través de una revolución cultural en la educación y en los valores de la sociedad.

\section{Justificación de lo sustentable}

La definición de la palabra sustentable involucra diversos aspectos muy importantes, entre los cuales podemos contemplar:

- La sustentabilidad tiene que ver con lo finito y delimitado del planeta, así como con la escasez de los recursos de la tierra.

- Con el crecimiento exponencial de su población.

- Con la producción limpia, tanto de la industria como de la agricultura.

- Con la contaminación y el agotamiento de los recursos naturales.

Los efectos de la interacción de estos fenómenos tienen varias implicaciones: de un lado, los recursos naturales, las materias primas y la energía que se utilizan en los procesos productivos, se explotan más rápidamente de lo que puedan restablecerse. De otro, la industria y la agricultura utilizan energías provenientes de recursos no renovables 


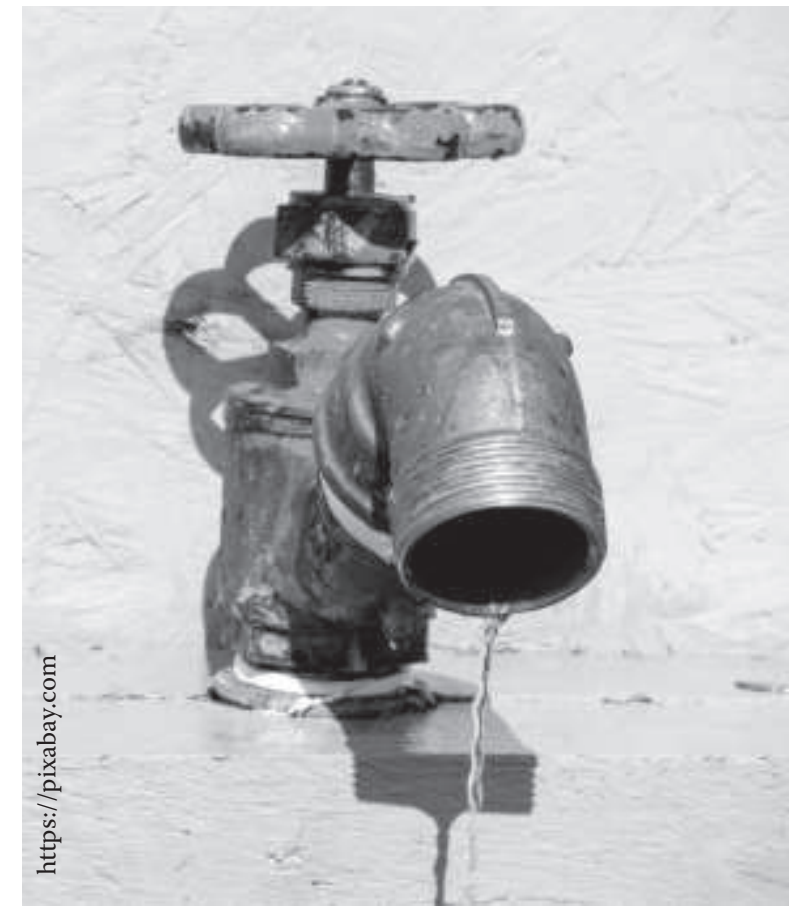

(carbón, petróleo, gas, etc.), y hoy en día se agota la capacidad natural del planeta de absorción de gases que producen el efecto invernadero para liberarse de los contaminantes generados por las prácticas actuales de energías contaminantes que se utilizan (Meadows, 1972).

Un sistema de vida donde la capacidad de alojamiento de los seres vivos sobre la tierra es siempre ascendente, acompañada de unos recursos agotables ante las necesidades crecientes de la población, aunado esto a un crecimiento económico con base en tecnologías contaminantes y consumidoras de mucha energía (que generan miles de millones de toneladas de desechos que se añaden al ecosistema cada año), conduce de esta manera a que indiscutiblemente la vida sobre la tierra tienda a colapsar, deteriorando la calidad de la vida humana, porque se rebasa la capacidad de equilibrio natural de los ecosistemas que la sustentan.

En otros términos, el concepto de sustentabilidad facilita entender que estamos ante un mundo con recursos naturales escasos y necesidades ilimitadas, una población siempre creciente, un desarrollo económico que ha venido dándose con base en tecnologías ya obsoletas con mucho consumo de energía generadoras de desechos contaminantes. Todo este panorama que está ya generando efectos climáticos devastadores nos ha llevado a comprender que existe una capacidad límite de sustentación para el planeta, y que nos estamos acercando rápidamente al colapso del ecosistema.

De otro lado, la sustentabilidad nos permite tener una visión, en la cual el tiempo juega un papel muy importante; por tanto, lo sustentable también está relacionado con una dimensión temporal, vinculando la correlación entre los hombres con el tiempo y la existencia de problemas para las generaciones futuras. De aquí que el Informe Brundtland considere que "...el desarrollo sostenible es el desarrollo que satisface las necesidades de la generación presente sin comprometer la capacidad de las generaciones futuras para satisfacer sus propias necesidades" (ONU, 1988, p. 67), es decir, nos está planteando que el planeta tierra que éstas generaciones estamos dejando, tiene que ser administrado en forma racional para las generaciones que vienen. Es decir, nos plantea una relación a través del tiempo, la relación existente entre la solidaridad intra-generacional con la solidaridad inter-generacional.

\section{Historia e ideas del concepto sostenible y/o sustentable}

\section{A. Orígenes}

En la década de los sesenta empieza a generarse la preocupación a nivel internacional por el tema del medio ambiente: importantes programas de gobierno y organismos internacionales, así como bastantes ensayos e informes de gran divulgación, estuvieron dedicados a la protección del medio ambiente con fines principalmente conservacionistas. Igualmente en dichos años se considera el surgimiento del movimiento ecologista y/o ambientalista contemporáneo, proponiendo cambios importantes a nivel político, social, cultural y económico; pero serían las dos grandes 
ONG con mayor entusiasmo en el estudio de estos temas, como fueron el Club de Roma y la Organización de las Naciones Unidas, quienes jugarían un papel importante, superando el tema de la protección del medio ambiente y llevándolo al debate de la crisis ambiental, considerándolo como agenda global de la comunidad internacional y como un tema de política mundial, regional y local.

Fue en abril de 1968 cuando por invitación del Club de Roma se reunieron allí diversas personalidades científicas, educadoras, economistas, humanistas, industriales y funcionarios nacionales e internacionales procedentes de 10 países, para discutir sobre el presente y el futuro de la especie humana. Se trataba de abordar las cuestiones que preocupan a todos los seres humanos: la pobreza en medio de la abundancia, la degradación del medio ambiente, el descrédito de las instituciones, la urbanización descontrolada, la incertidumbre en el empleo, entre otros aspectos (Mayor Zaragoza, 2000).

Por su parte, la ONU celebra en París, en aquél mismo año, la Conferencia sobre la Conservación y el Uso Racional de los Recursos de la Biosfera, cuyo propósito fue el que los países convocados asumieran la responsabilidad internacional con relación al medio ambiente del planeta y se promoviera un encuentro mundial sobre el Medio Ambiente Humano, que se concretaría en 1972.

En marzo de 1972, Universe Books de Nueva York, publica el libro Los Límites del Crecimiento (The Limits to Growth), estudio contratado por el Club de Roma al equipo del MIT (Massachussets Institute of Technology), dirigido por el profesor Dennis Meadows y realizado por Donella $\mathrm{H}$. Meadows, Dennis L. Meadows, Jorgen Randers y William W. Behrems, los cuales presentan allí los cinco factores básicos que determinan, y en último término limitan, el crecimiento en el planeta Tierra: población, producción agrícola, recursos naturales, producción industrial y contaminación (Meadows, 1972).

Igualmente, del 5 al 16 de junio de 1972 se celebra la Conferencia de las Naciones Unidas sobre el Medio Humano en el país de Suecia, también conocida como la Conferencia de Estocolmo, cuyo tema central era inspirar y guiar a los pueblos del mundo en la preservación y mejora del medio ambiente humano, a partir de considerar las necesidades sociales y culturales de planificar la protección ambiental, los recursos naturales y sobre los medios a emplear internacionalmente para luchar contra la contaminación. De éste año en adelante, Las Naciones Unidas realizarán periódicamente conferencias internacionales reseñándose las principales sintéticamente en el anexo del presente ensayo.

En el año de 1984 se reunió por primera vez la Comisión Mundial de Medio Ambiente y Desarrollo de la ONU, con el convencimiento que era posible para la humanidad construir un futuro más próspero, más justo y más seguro, y entre cuyos objetivos estaban los de examinar los temas críticos de desarrollo económico y medio ambiente y formular propuestas realistas al respecto. Pero será hasta 1987 cuando dicha comisión presenta su "Informe Brundtland", llamado así en honor a la Secretaria de las Naciones Unidas de ese entonces, la activista europea Gro Harlem Brundtland (ONU, 1988).

Es en este libro, el concepto en consideración gana reconocimiento a nivel internacional, clarificando que "...el desarrollo sostenible es el desarrollo que satisface las necesidades de la generación presente sin comprometer la capacidad de las generaciones futuras para satisfacer sus propias necesidades" (ONU, 1988, p. 67).

Para abril de 1990, se realiza en Washington la conferencia anual sobre Desarrollo Económico del Banco Mundial, donde el economista holandés Peter Nijkamp presenta el trabajo titulado "Regional Sustainable Development and Natural Resources Use" traducido como "Desarrollo Regional Sustentable y el uso de Recursos Naturales", donde sintetiza el concepto de sustentabilidad, representando gráficamente la relación entre el crecimiento económico, la equidad social y la sustentabilidad ambiental para dar lugar al desarrollo sustentable, área central del denominado triángulo de Nijkamp. 
Gráfico 1. Triángulo de Nijkamp

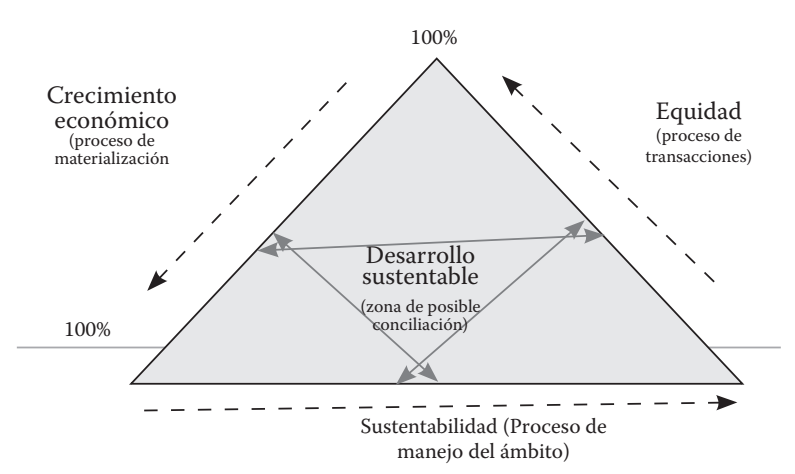

Hipotéticamente, el desarrollo sustentable se logra cuando los tres objetivos son simultáneamente alcanzados, tal y como se puede ver en el triangulo de Nijkamp, representando la armonía entre el crecimiento económico, la equidad social y la sustentabilidad ambiental, área central del triangulo.

En septiembre de 2000 se acuerda en la sede de las Naciones Unidas en Nueva York la Cumbre del Milenio de las Naciones Unidas, cuyo objetivo era reunir esfuerzos para combatir la pobreza, el hambre, las enfermedades, el analfabetismo, la degradación del medio ambiente, la discriminación contra la mujer y crear una asociación mundial para el desarrollo, Declaración conocida como los 8 objetivos del Desarrollo del Milenio. (Naciones Unidas, 2000).

En el año 2005 comienza la década de la Educación para la Sostenibilidad promovida por las Naciones Unidas, con el objetivo de impulsar la necesaria transición hacia la sostenibilidad. Conveniente resaltar que cumplidos estos diez años, nuevamente se impulsa el compromiso internacional de fomentar la educación para el desarrollo sostenible a partir del Programa de Acción Global (GAP por sus siglas en inglés, Global Action Programme).

En 2015, las Naciones Unidas deciden transmitir el documento final titulado "Transformar nuestro mundo: la Agenda 2030 para el Desarrollo Sostenible". Dicha nueva Agenda Universal contempla los 17 Objetivos del Desarrollo Sostenible, pretendiendo retomar, por un lado, los Objetivos del
Desarrollo del Milenio y lograr lo que con ellos no se consiguió, así como, contribuir a la construcción de un futuro sostenible: "Estamos resueltos a liberar a la humanidad de la tiranía de la pobreza y las privaciones, y a sanar y proteger nuestro planeta. Estamos decididos a tomar las medidas audaces y transfor- mativas que se necesitan urgentemente para reconducir al mundo por el camino de la sostenibilidad y la resiliencia". Importante señalar que dicha iniciativa surge como respuesta al Acuerdo alcanzado en la Cumbre de la Tierra Río+20, celebrada en junio de 2012 en Río de Janeiro.

Resulta substancial destacar que las Naciones Unidas en la Declaración del 12 de agosto del 2015, cuando hacen referencia a "Nuestros Principios y Compromisos Comunes", resumen en el inciso 11 la importancia que ha tenido el tema de la sostenibilidad en los últimos 20 años cuando señalan y reafirman:

"Los resultados de todas las grandes conferencias y cumbres de las Naciones Unidas, que han establecido una base sólida para el Desarrollo Sostenible y han ayudado a conformar la nueva Agenda, como la Declaración de Río sobre el Medio Ambiente y el Desarrollo, la Cumbre Mundial sobre el Desarrollo Sostenible, la Cumbre Mundial sobre Desarrollo Social, el Programa de Acción de la Conferencia Internacional sobre la Población y el Desarrollo, la Plataforma de Acción de Beijing y la Conferencia de las Naciones Unidas sobre el Desarrollo Sostenible” (ONU, 2015).

\section{B. Las ideas sobre la sostenibilidad y/o sustentabilidad}

Para algunos autores, es a partir del informe Brundtland que se acota el término inglés "sustainable development" como desarrollo sostenible. De allí la confusión entre si existe o no diferencia alguna entre los términos "desarrollo sostenible" y "desarrollo sustentable". La única diferencia entre estos dos términos viene de su traducción desde el inglés, que en el caso mexicano fue traducido como desarrollo sostenible mientras que en otros 
países de habla hispana el término resultó ser el de desarrollo sustentable. La misma idea se encuentra en el libro De la economía a la ecología (Riechmann, 1995, p. 7).

Para otros, la esquematización del triangulo de Nijkamp dará lugar a una libre interpretación dependiendo de lo que se entienda sobre qué es lo económico, lo social y lo ambiental; es decir, dependiendo de la concepción que se tenga de cada uno de estos tres objetivos y/o su relación, así mismo se definirá la comprensión de qué es la sostenibilidad y/o sustentabilidad ó de qué concepto se está hablando.

Igualmente es importante señalar que de aquí en adelante, los términos de la sostenibilidad y la sustentabilidad se utilizan bien como sinónimos o inequívocamente, dependiendo de las tendencias ideológicas y/o de los intereses propios de quien lo profese, por cuanto el término sostenible, hoy en día, es aceptado universalmente por la mayoría de las personas.

Los anteriores hechos han distorsionado el concepto de sustentabilidad en varios sentidos, como por ejemplo el de asociar el crecimiento sostenido con el crecimiento sostenible y por deducción con el desarrollo sostenible. La erosión semántica queda explícita, por cuanto la expresión crecimiento sostenido hace referencia al crecimiento económico a lo largo del tiempo, es decir, a la tasa de crecimiento del PIB exclusivamente; mientras que el desarrollo sustentable es un proceso armonioso entre las distintas disciplinas del conocimiento, especialmente en lo económico, social, ambiental, cultural y/o a un sistema de valores correspondiente.

La distorsión semántica de lo sostenible se acrecienta y se presenta muy ambigua, cuando dicho concepto es aplicado indistintamente a la producción, la ecología, la economía y el medio ambiente (Dourojeanni, 2000, p. 22).

Es frecuente encontrar en el pénsum de las universidades, carreras asociadas exclusivamente a la sostenibilidad -diseño sostenible; turismo sostenible; sostenibilidad ambiental, etc.-, y en la amplia literatura técnica de lo sostenible, mencionar el término sostenible y la posibilidad existente de encontrar bien sea la sostenibilidad económica, la sostenibilidad social o la sostenibilidad ambiental, lo cual permite intuir que es posible conseguir el desarrollo sostenible económico, el desarrollo sostenible social y el desarrollo sostenible ambiental, por separado, independientemente de la interacción de dichos ejes, los cuales conforman la sustentabilidad.

Evidentemente, el desarrollo económico sostenible puede lograrse, por ejemplo, cuando las empresas o personas, con un criterio rentable, hacen posible que sus proyectos sean financieramente exitosos, (lograr en el tiempo que los ingresos sean mayores que sus costos); sin importar que al mismo tiempo abran su capital financiero a muchos socios, tengan un trato digno con los trabajadores y sus clientes, $\mathrm{e}$ inclusive, paguen buenos salarios a la comunidad. Un ejemplo de ello, son algunas de las empresas cuyo razón social se relaciona con la responsabilidad social.

Por su parte, la sostenibilidad social puede conseguirse cuando se apoyan proyectos en el mantenimiento de la cohesión comunitaria, para el emprendimiento de objetivos comunes alrededor de mejorar las condiciones de vida. Un ejemplo de ello son las ONG's sociales y ambientalistas.

La sostenibilidad ambiental se obtendrá siempre y cuando la explotación de los recursos naturales se mantenga dentro de los límites de la regeneración y el crecimiento natural, a partir de planear la explotación de los recursos y de precisar los efectos que la explotación tendrá, sobre el conjunto del ecosistema.

Un ejemplo de ello es la denominada economía verde, cuyo propósito es incidir en la producción de proyectos verdes (derivados de inversiones estratégicas tanto públicas como privadas), la cual considera a la tierra como la infraestructura para la vida que depende de su sistema bioproductivo, generando beneficios globales a partir de la preservación y conservación de los ecosistemas; por tanto, es posible tener sociedades sostenibles sin 
perjudicar el medio ambiente y vivir de la explotación que éste proporcione.

Igualmente la economía verde postula el desarrollo de tecnologías respetuosas con el medio ambiente, con tecnologías limpias, a partir de incentivos económicos hacia los mercados verdes, conservando los ecosistemas bioproductivos en la tierra, cuyas ventajas serían la disminución de la contaminación ambiental. Es importante señalar que en Río+20 (2012), fue posible conseguir que los 193 países participantes se comprometieran a adoptar el concepto de la economía verde (ONU, 2000; ONU, 2012).

De igual manera, la economía azul plantea el logro de la sostenibilidad ambiental, a partir del respeto por los recursos naturales, aprovechando al máximo las materias y la energía disponible, basadas en las leyes de la física y en la fuerza de la gravedad como fuente principal de energía (Pauli, 2011).

El concepto de desarrollo sostenible se ha propagado y enriquecido, pero también se ha fragmentado, separándose de su origen. Me atrevo a asegurar que dichos conceptos que nacieron como idénticos, se han divorciado y a la larga se irán distanciando cada día más.

Sin duda alguna, Nuestro Futuro Común (ONU, 1988) originó una gran atención en los medios científicos, académicos, empresariales, movimientos ambientalistas y en la comunidad en general, constituyéndose hoy en el referente y paradigma para el estudio de la sustentabilidad.

Este grandioso documento, después de analizar los capítulos referentes al desafío mundial; las direcciones de la política; la cooperación internacional y las reformas de las instituciones; así como el llamamiento a la acción,
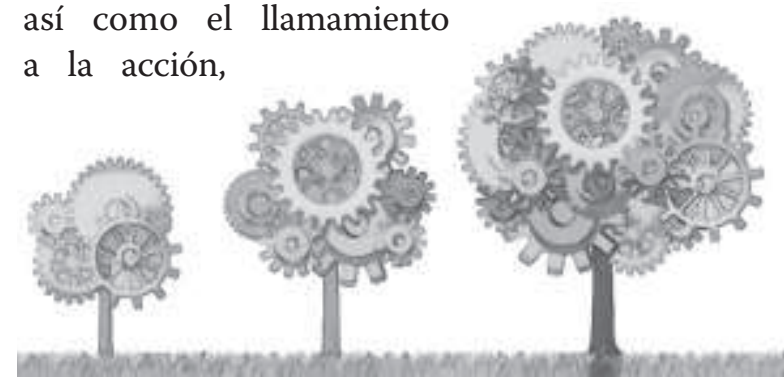

permite entrever la dificultad para conciliar el crecimiento económico con la eliminación de la pobreza y el mejoramiento de las condiciones del medio ambiente, dado que el estilo de desarrollo dominante tiende a exacerbar más que a atenuar los desequilibrios económicos, distributivos y ambientales prevalecientes. Con este mensaje se aclara también la importancia de la relación directa existente entre las dimensiones de lo económico, lo social y el medio ambiente.

Como ya lo habíamos anotado, es allí donde gana reconocimiento a nivel internacional el concepto de desarrollo sostenible, definiéndolo como “... asegurar que satisfaga las necesidades del presente sin comprometer la capacidad de las futuras generaciones para satisfacer las propias" (ONU, 1988, p. 29); asimismo , allí , se llama la atención que el objetivo de lo sostenible es la satisfacción de las necesidades humanas (garantizando el alimento, el trabajo, la vivienda y el abrigo, entre otros) y la consecución de las aspiraciones humanas entendidas como "mejorar la calidad de vida para tener una vida mejor", para lo cual se requerirá una mayor redistribución equitativa de los recursos, mayores niveles de productividad y un cambio sustancial a nivel tecnológico, con la implantación de tecnologías limpias en los diversos sectores económicos, sin explotar en exceso los recursos naturales y sin afectar el medio ambiente o afectándolo de manera moderada, manejable.

Conscientes que el crecimiento económico trae importantes cambios en los ecosistemas físicos, dicho informe, llama la atención acerca de la importancia que los recursos renovables no se agoten (montañas, páramos, bosques, ríos, bancos de peces, etc), para lo cual la explotación de los recursos naturales se debe mantener dentro de los límites de la regeneración y el crecimiento natural, a partir de planear la explotación de los mismos y de precisar los efectos que la explotación tendrá sobre el conjunto del ecosistema.

Este es el verdadero concepto que nació como sostenible pero, por razones anteriormente expresadas, es preferente denominarlo como sustentable. 
Como colofón de este ensayo pudiéramos presentar dos conceptos bastante definidos que si bien tienen aristas comunes necesariamente se deben demarcar claramente como son: cuando lo sostenible hace relación entre la armonía existente entre lo económico, lo social, lo ambiental es sinónimo de lo sustentable, a diferencia de lo sostenible cuando se consigue cada uno de dichos ejes por separado.

Dejo así en estos términos plasmadas mis apreciaciones como un modesto aporte a la discusión válida de quienes quieran aportar sus puntos de vista y lo respalden dentro de un marco de dinámica crítica al desarrollo del conocimiento.

\section{La esencia de la sustentabilidad está en lo transversal}

La sustentabilidad es un concepto integrador valioso, por cuanto se adapta a cualquier lugar geográfico que se esté analizando, se adecúa a los diferentes objetivos que se estén considerando, tiene en cuenta las presentes y futuras generaciones, pero sobre todo, retoma la necesidad nuevamente de concebir al hombre como parte integrante de la biosfera.

Si se considera que el crecimiento económico es la capacidad de un país en proporcionar a su pueblo bienes y servicios apropiados a sus necesidades, donde se establezca una relación no-destructiva con la naturaleza y se promueva la equidad entre las diferentes clases sociales. La equidad social, concebida como el fortalecimiento de un estilo de desarrollo que no perpetúe ni profundice la pobreza ni, por tanto, la exclusión social, sino que tenga como uno de sus objetivos centrales la erradicación de aquélla y la justicia social, y la participación social en la toma de decisiones, es decir, que las comunidades y la ciudadanía se apropien y sean parte fundamental del proceso de desarrollo, y que lo ambiental, esté referido a la necesidad que el impacto del proceso de desarrollo socio-económico no destruya de manera irreversible la capacidad de carga del ecosistema.
Entonces, la sustentabilidad en sentido amplio, puede ser entendida como la producción de bienes y servicios, donde se satisfagan las necesidades humanas y se garantice una mejor calidad de vida a la población en general, con tecnologías limpias en una relación no destructiva con la naturaleza, en la cual la ciudadanía participe de las decisiones del proceso de desarrollo, fortaleciendo las condiciones del medio ambiente y explotando los recursos naturales, dentro de los límites de la regeneración y el crecimiento natural.

A nivel práctico puede hablarse de un desarrollo sustentable cuando, desde el punto de vista económico, se pueda admitir que no hay mejor incentivo en la vida que un trabajo estable y bien remunerado, donde se genere empleo a través de proyectos comunitarios de emprendimiento con responsabilidad en el consumo y en la elaboración de tecnologías que eliminen los clorofluorocarbonos y los gases de efecto invernadero; lo social esté relacionado con la creación de un tejido social con empoderamiento de su territorio a través de una cultura ciudadana; y lo ambiental sea coherente con la formación cultural y/o sistema de valores, preservando y conservando los bienes naturales, e inmateriales de la sociedad.

No obstante la clave de la sustentabilidad está en la transversalidad, es decir, en los desarrollos comunes entre los subsistemas considerados que constituyen el progreso de un lugar y/o territorio específico, regulando el avance del hombre con su entorno y estableciendo una relación armoniosa entre lo económico, lo social, lo ambiental, lo cultural y/o el sistema de valores.

De esta manera, la sustentabilidad debe entenderse como una disciplina articulada del conocimiento y como una nueva manera de repensar la relación de los hombres con la naturaleza, a partir de la integralidad de las dimensiones económicas, sociales, ambientales y de valores, que conlleve a una revolución global de supervivencia con el planeta.

¿Será posible considerar, hoy en día, una sociedad sustentable bajo las actuales condiciones del sistema económico? Este interrogante me motiva 


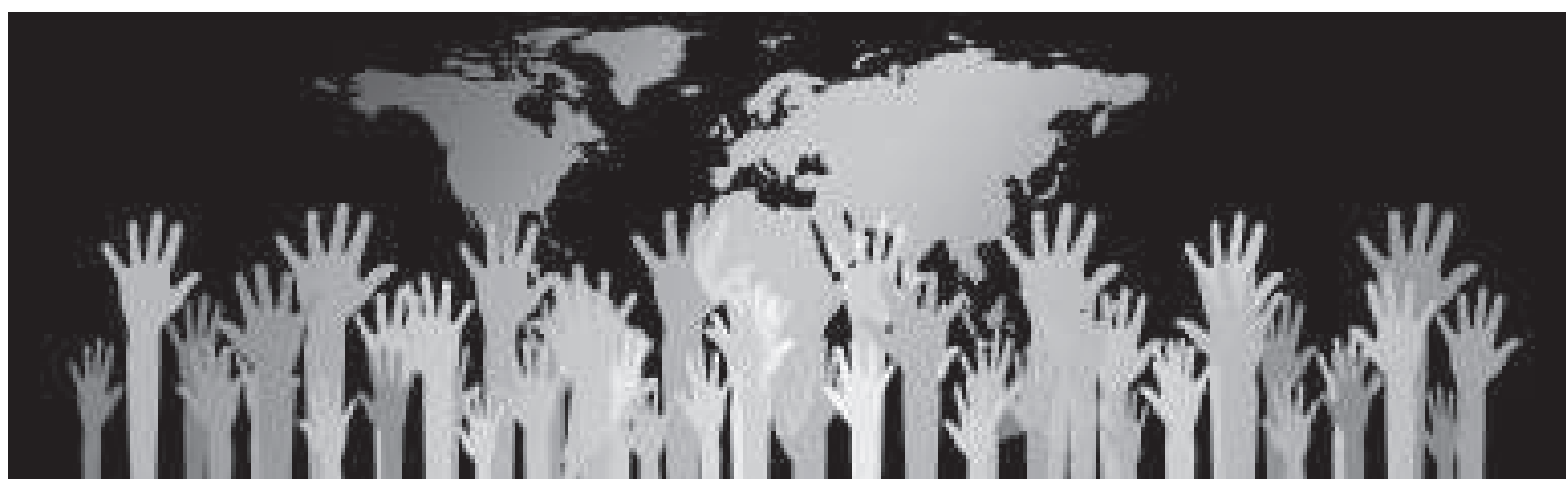

http://fundacionoxigenoglobal.org/

a seguir estudiando este tema, para presentarles posteriormente mis opiniones, y someterlas a consideración de los lectores, hasta lograr la dinámica de la discusión que amerita el apasionamiento entre lo académico, lo cognoscitivo y lo sensorial en la vida que llevamos.

\section{Anexo}

\section{Protocolo de Montreal 1987}

Su propósito fue la eliminación de las emisiones mundiales de sustancias agotadoras de la capa de ozono (SAO). El Protocolo propone la reducción en su producción y consumo hasta llegar a la eliminación parcial.

\section{Cumbre de la Tierra Río de Janeiro}

\section{-Agenda 21 de 1992}

Necesidad de disminuir los gases que causan el efecto invernadero; se aborda la problemática que tienen los bosques y las selvas en Sudamérica y se tomo en consideración la recuperación de la diversidad biológica en el mundo.

\section{Protocolo de Kyoto 1997}

$\mathrm{Su}$ pretensión principal fue reducir los gases que causan el calentamiento global, estos gases son: Dióxido de carbono $\left(\mathrm{CO}_{2}\right)$, Gas metano $\left(\mathrm{CH}_{4}\right)$ y Óxido nitroso $\left(\mathrm{N}_{2} 0\right)$. Además de tres gases industriales fluorados: Hidrofluorocarbonos (HFC),
Perfluorocarbonos (PFC) y Hexafluoruro de azufre $\left(\mathrm{SF}_{6}\right)$.

\section{Conferencia de Estocolmo -2001}

Se determina la existencia de sustancias toxicas que debían ser reguladas e incluso eliminadas; entre las más comunes se encontraban: Insecticida, ectoparasiticida locales, termiticida, aditivo para adhesivos de contrachapado, productos agrícolas, todos ellas denominadas Contaminantes Orgánicos Persistentes -COPs-.

\section{Cumbre de la Tierra de Johannesburgo $-2002$}

El objetivo principal era evaluar todo lo que se había planteado en la anterior cumbre de la tierra. Igualmente se trató el Desarrollo Sostenible, su objetivo era la adopción de un plan de acción de 153 artículos divididos en 615 puntos sobre diversos temas: la pobreza y la miseria, el consumo, los recursos naturales y su gestión, globalización, el cumplimiento de los Derechos humanos; uno de los puntos fuertes en esta cumbre fue el derroche de energía que tienen algunos países.

\section{Río de Janeiro +20 -2012}

Uno de los principales logros de la cumbre fue el hecho de que los 193 países participantes se comprometieron a adoptar el concepto de la Economía Verde. Igualmente se ponen en consi- 
deración los Objetivos de Desarrollo Sostenible (ODS): son 17 metas que los Gobiernos se impondrán para asuntos vitales como el agua, la tierra y la biodiversidad, están inspirados en los Objetivos de Desarrollo del Milenio (ODM). Se proponen metas para temas como el hambre, pobreza, educación y salud, los cuales se comprometieron a mejorar sus índices todos los países para 2015.

\section{Paris Cop21 -2015}

Tiene como objetivo mantener la temperatura media mundial muy por debajo de 2 grados centígrados respecto a los niveles preindustriales, aunque los países se comprometen a llevar a cabo todos los esfuerzos necesarios para que no rebase los 1,5 grados y evitar así impactos catastróficos. El acuerdo adoptado es legalmente vinculante, pero no la decisión que lo acompaña ni los objetivos nacionales de reducción de emisiones. No obstante, el mecanismo de revisión de los compromisos de cada país sí es jurídicamente vinculante para tratar así de garantizar el cumplimiento.

Con respecto a la reducción de emisiones, 187 países de los 195 que han participado en la COP21 han entregado sus compromisos nacionales de lucha contra el cambio climático que entrarán en vigor en 2020.

\section{Bibliografía}

Dourojeanni, A. (2000). Procedimientos de Gestión para el Desarrollo Sustentable. Santiago de Chile: Naciones Unidas - CEPAL - ECLAC.

Mayor Zaragoza, F. (2000). Los Limites del Crecimiento. Tribuna Libre, 5 .

Meadows, D. H. (1972). Los Límites del Crecimiento. Mexico: Fondo de Cultura Económico.

ONU (2015). Asamble General. Nueva York: Naciones Unidas.

ONU (2000). Asamblea General Cumbre del Milenio. Nueva York.

ONU (1988). Nuestro Futuro Común. Madrid: Alianza Editorial.

Pauli, G. (2011). La Economía Azul. Madrid: Tusquets Editores.

Riechmann, J. (1995). De la economía a la ecología. Madrid: Trotta. 